\title{
Comparison of MIC with MBEC Assay for in Vitro Antimicrobial Susceptibility Testing in Biofilm Forming Clinical Bacterial Isolates
}

\author{
Summaiya Mulla1, Ambuj Kumar², Sangita Rajdev ${ }^{1 *}$ \\ ${ }^{1}$ Government Medical College, Surat, India \\ ${ }^{2}$ University of South Florida, Tampa, FL, USA \\ Email: mullasummaiya@gmail.com, akumar1@health.usf.edu, "drsangitarajdev@gmail.com
}

Received 10 December 2015; accepted 13 February 2016; published 18 February 2016

Copyright (C) 2016 by authors and Scientific Research Publishing Inc.

This work is licensed under the Creative Commons Attribution International License (CC BY). http://creativecommons.org/licenses/by/4.0/

\section{(c) (i) Open Access}

\section{Abstract}

Context: MIC results can be misleading for treatment of biofilm associated. The Minimum Biofilm Eradication Concentration (MBEC) measures the determination to be made for a biofilm susceptibility to antibiotics. Aims: Assessment of biofilm production and comparison of the MIC and MBEC assays evaluate differences in the antibiotic sensitivity patterns of different clinical bacterial isolates from patients implanted with medical devices. Settings and Design: Random sampling with experimental study at tertiary care institute. Methods and Material: The study was carried out during January 2014 to March 2014 on 50 positive bacteriological cultures of medical devices which were inserted in hospitalized patients. Biofilm forming strains were identified by tissue culture plate method \& tube method. Biofilm-producing and non-biofilm forming reference strains were used as controls. Assay has been developed for the use with flat bottom, 96-well microtiter plates. Sterile autoclaved PCR tubes were used as pegs which provided surface for the biofilm formation. Amikacin, ciprofloxacin, trimethoprim-sulfamethoxazole, vancomycin, cefoperazone/ sulbactam, gentamycin were tested for MIC and MBEC assay. Statistical Analysis Used: Results will be discussed in the form of percentages. Results: Colonization by Klebsiella pneumoniae, Acinetobacter baumanni and Pseudomonas aeruginosa was prevalent bacterial isolates in medical devices. MBEC was higher for all the antibiotics as compared to MIC except amikacin MBEC for Pseudomonas was the same as MIC. Conclusions: Device associated bacterial biofilms are the major source of infections in patients of critical care setup. MIC misleads physician for organism's drug susceptibility testing, which results in therapeutic failure. MBEC can guide regarding choice and proper dosing of antibiotics to be given. That's why major studies for similar testing should be done with clinical evaluation.

\footnotetext{
Corresponding author.
} 


\section{Keywords}

\section{Bacterial Biofilms, Antibiotics, MIC, MBEC}

\section{Introduction}

Medical devices are frequently inserted in ICU patients for various interventions and it's in continuous contact with body fluids and irrigation fluids. If it gets implanted with bacteria then it serves as an excellent surface for the formation of bacterial biofilms. This colonization of bacterial biofilms can be reservoir of bacteria and the source of infection [1]. Bacteria in biofilm communities behave differently as compared to planktonic form. Bacteria in biofilm community are generally highly drug resistant because of increased production of extracellular matrix, multi-layered colonies, decreased metabolic rates, decreased multiplication and polymicrobial colonization. Ultimately it becomes good source of infection in patients with poor immunity with drug resistant bacteria. These phenotypic changes are recognized as a cause for frequent treatment failure in patients implanted with infected medical devices and prosthetic materials [2] [3]. Routinely ant microbiology laboratory evaluates susceptibility of bacteria towards any antibiotics by either disc diffusion test or MIC testing. Susceptibility of bacteria in biofilms cannot be evaluated by such testing methods and requires some special system of testing. The Minimum Biofilm Eradication Concentration (MBEC) measures the susceptibility of specified antibiotic for a biofilm. The Clinical Laboratory Standards Institute (CSLI) provides testing of bacteria in plantonic form and does not have method for antimicrobial susceptibility testing against biofilm associated organisms. However, a variety of protocols for testing antimicrobial agents against biofilms have been suggested. Ceri et al. developed the MBEC assay and used it to test several antimicrobial agents against biofilms [4] [5].

This study was planned to develop an in vitro model system to compare the MIC and MBEC assays to evaluate differences in the antibiotic sensitivity patterns of different isolates from patients implanted with medical devices.

\section{Subjects and Methods}

The present study assesses antibiotic susceptibility of bacterial biofilm which was conducted in microbiology laboratory at new civil hospital, Surat after having ethical clearance from institute review board. Samples were tested obtained from Medical ICU, Neonatal ICU and other ward/units. Varied type of medical devices were received for testing like endotracheal tube, tracheostomy tube, Foley's catheter, CVP tip, Pigtail catheter etc. The study was carried out during January 2014 to march 2014 on 50 positive bacteriological cultures of medical devices which were inserted in hospitalized patients from different units and wards.

Biofilm formation assay: Analysis of biofilm formation was performed by tissue culture plate method as well as tube method.

Tissue culture plate method (TCP)/Microtitre plate method: Isolates were inoculated in tryptic soy broth (TSB) with $0.25 \%$ glucose; density was standardized by comparison with $0.5 \mathrm{McF}$ arland standards (i.e. $10^{8} \mathrm{CFU} / \mathrm{ml}$ ) and incubated for 24 hour at $37^{\circ} \mathrm{C}$. At the end of incubation period, cultures were diluted 1:100 with fresh media. Controls were set with biofilm producing reference strains of Acinetobacter baumanni (ATCC 19606) (Kwikstik, France) and Pseudomonas aeruginosa (ATCC 27853) (Kwikstik, France) and non-biofilm forming reference strain of Staphylococcus aureus (ATCC 25923) (Kwikstik, France) and E. coli (ATCC 25922) (Kwikstik, France). Uninoculated broth was also used in microtitre plate method serving as control to check sterility and non-specific binding of media. The cut-off was defined as three standard deviations above the mean ODc. Each isolate was classified as follows: weak biofilm producer OD $=2 \times$ ODc, moderate biofilmproducer $2 \times$ ODc $<$ OD $=4 \times$ ODc, or strong biofilm producer OD $>4 \times$ ODc [6]-[9].

Antimicrobial sensitivity: biofilm vs. Planktonic cells (using the MBEC assay): Assay has been developed for the use with flat bottom, 96-well microliter plates. Sterile autoclaved PCR tubes were used as pegs which provided surface for the biofilm formation.

Biofilm Formation: For inoculum preparation, bacterial colonies were collected from agar plate and dissolved in growth medium (TSB with $0.25 \%$ ) and density of suspension was standardized by comparison with 1.0 McFarland standard (i.e. $3.0 \times 108 \mathrm{cfu} \cdot \mathrm{ml}^{-1}$ ). Culture was diluted at 1:30 in fresh growth medium to give an ap- 
proximate count of $1.0 \times 107 \mathrm{cfu} \cdot \mathrm{ml}^{-1}$ into sterile polypropylene or glass tube serves as the inoculums. $150 \mu \mathrm{l}$ of the 1 in 30 dilution was added to each well of the 96-well microtiter plate. Autoclaved pegs were inserted in growth media. Plate was covered by aluminum foil and incubated in a humidified incubator at the appropriate temperature. Biofilm formation was confirmed by staining of one peg with $0.1 \%$ safranine for 30 min as shown in Figure 1.

Antibiotic Susceptibility: In this study we have taken amikacin, ciprofloxacin, trimethoprim-sulfamethoxazole, vancomycin, cefoperazone/sulbactam, and gentamycin for MIC and MBEC assay. Stock solutions of $5 \times$ the highest concentration antibiotics were prepared and stored at $-80^{\circ} \mathrm{C}$. Working solution was prepared by diluting stock solution 1:5 by adding Cation Adjusted Muller Hinton Broth (CAMHB, himedia, Mumbai).

Biofilm Susceptibility testing: Biofilms formed on the surface of pegs were transferred to a standard 96-well plate as shown in Figure 2 in which required dilutions of different antibiotics were prepared with Cation Adjusted Muller Hinton Broth (CAMHB, himedia, Mumbai). Antibiotic plate was incubated overnight at $37^{\circ} \mathrm{C}$ and after that pegs were removed, rinsed in PBS (pH: $7.2 \pm 0.2$ ) and MIC determination was done. MIC was determined, by checking turbidity visually in the wells of challenge plate and alternatively, it was determine Optical Density (OD) with a micro ELISA auto reader at wavelength of $450 \mathrm{~nm}$ with $630 \mathrm{~nm}$ reference ranges. Biofilm disruption was done by transferring pegs in second plate containing Cation Adjusted Muller Hinton Broth (CAMHB, himedia, Mumbai). Mechanical disruption of biofilms were done by simply shaking the plate on shaker for 2 minutes and then incubated at $37^{\circ} \mathrm{C}$ for 24 hrs. MBEC was determined, by checking turbidity visually in the wells of recovery plate and alternatively, it was determine Optical Density (OD) with a micro ELISA auto reader at wavelength of $450 \mathrm{~nm}$ with $630 \mathrm{~nm}$ reference ranges [8].

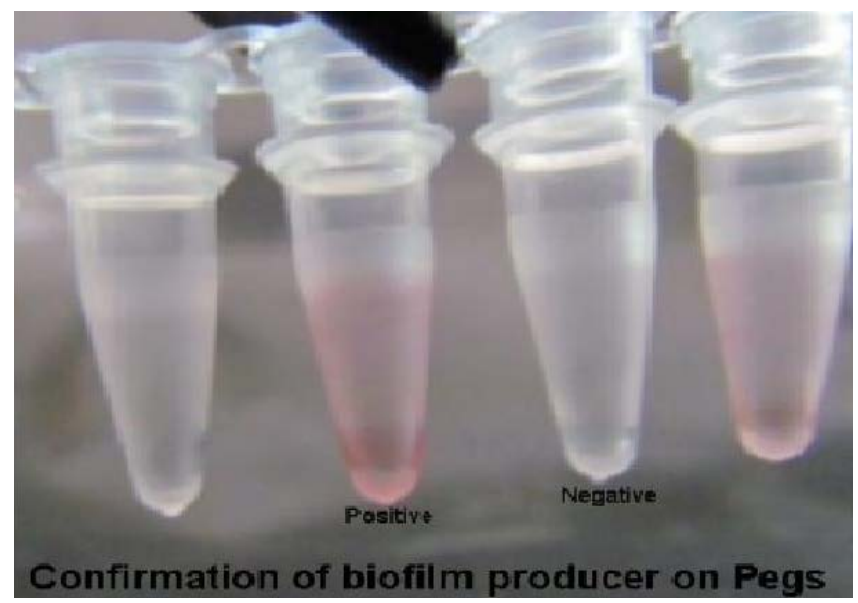

Figure 1. Pegs showing stained biofilms on outer surface.

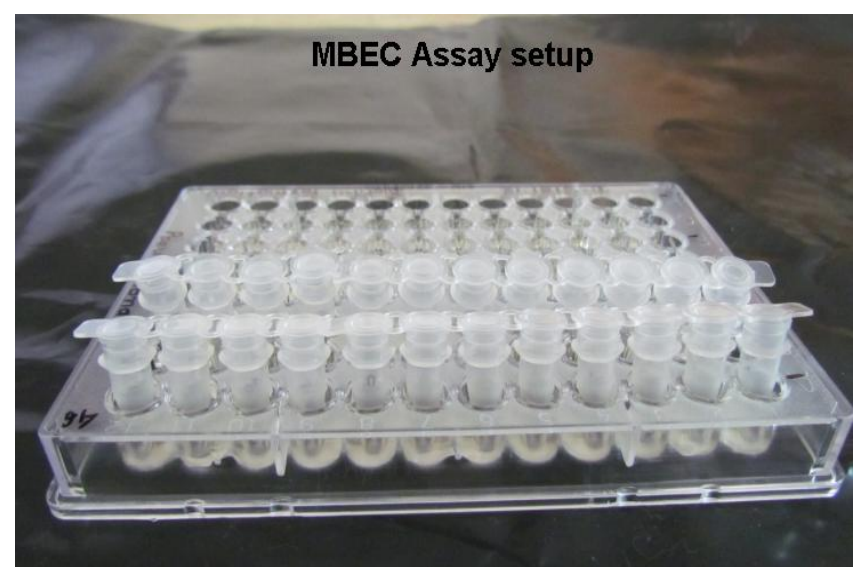

Figure 2. Novel system to check minimum biofilm eradication concentration MBEC of antibiotics against bacterial biofilms. 


\section{Results}

Bacterial biofilms possess high load of infection on patents of critical care unit and responsible for multidrug resistant nosocomial infections and also one of the cause of treatment failure. Biofilms have great significance for public health, because bacterial biofilms are everywhere and sometimes it's difficult to remove the source. That's why it's required to treat it with right dose of right drug.

To assess the burden of biofilm in our setup was the aim for this study. In this hospital high percentage of biofilm producing isolates were observed which had high resistant to antibacterial agents. Patients admitted in Medical ICU, Neonatal ICU, Surgery, Gynaecology and Paediatrics wards/units of this hospital were the source of specimen. Specimens in the form of medical devices were received from different wards/units of New Civil Hospital, Surat; total 50 samples were studied. All the isolates obtained from different indwelling medical devices were screened by standard TCP/micro titre plate method and Tube method for biofilm production. Among 50 isolates $60 \%$ biofilm producers detected by TCP method and 38\% by tube method. Distributions of different isolates are as shown in table No 1 . Out of 50 bacterial isolates studied, 13 were Acinetobacter baumanni, 12 were Klebsiella pneumoniae, 9 were Pseudomonas aeruginosa, 6 were E. coli, 2 were each of Staphylococcus aureus and coagulase negative staphylococci, 1 each of Proteus spp. and Enterobacter cloacae. Major medical devices received were Endotracheal tube 36, followed by Foley's catheter 5, CVP tip 4, tracheostomy tip 3 and SPC tip and pigtail catheter 1 each as shown in table no 1. Chart 1showed the quantitative assessment of biofilm production by different clinical bacterial isolates as number of weak, moderate or strong biofilm producers. Chart 2 showed the comparison between MIC and MBEC value of various antibiotics like cefoperazone/ sulbactam, gentamycin, amikacin \& ciprofloxacin. Higher amount of antibiotics were required to kill bacterial biofilms than planktonic form.

\section{Discussion}

Biofilm associated infections are generally recurrent and chronic. It's generally associated with treatment failure because of higher level of drug resistance. Biofilms can be visually inspected upon medical devices while removing from patient's body and should be cultured to know type of organism colonized so that early onset of therapy can be initiated. Some patients with septicaemia, just removal of device can't help so proper antibiotic coverage should be started keep in mind about nature of organism. In this study, it is observed that majority isolates were from MICU (35.3\%) stating that the highest use of interventional procedures with use of medical devices in MICU. Isolates were followed by NICU (21.6\%), Surgery Ward (13.7\%), Paediatric Ward (13.7\%), Medicine Ward (7.8\%) and Gynaecology Ward (7.8\%). Table 1 shows different medical devices colonised by different bacterial like endotracheal tube are the most common medical device used to such setups and it frequently gets colonization by Klebsiella, Acinetobacter and Pseudomonas as prevalent bacterial isolates, followed by E. coli, Staphylococci, Proteus, and Enterobacter. Endotracheal tube colonization and biofilm formation occurs in many patients undergoing mechanical ventilation, from a very early stage so proper antibiotic coverage is required to prevent infections in such patients. Enterococci are more commonly associated with colonization of Foley's catheter. Biofilm forming organism presents on indwelling medical devices causing recurrent infections.

Coagulase negative Staphylococci, Staphylococcus aureus and Proteus mirabilis formed biofilm in 100\% of bacterial isolates whereas Pseudomonas aeruginosa in 78\%, Acinetobacter baumannii in 77\%, Enterococcus faceium in 50\% and Klebsiella pneumoniae in 45\%, of bacterial isolates formed biofilm while Escherichia coli and Enterobacter cloacae did not form biofilm. In present study, tube method showed 2 false positive and 11 false negative results.

MIC and disc diffusion methods tests the susceptibility of bacteria in free form but bacteria behaves differently in biofilm communities. It requires special condition of invitro biofilm development and then challenges of different antibiotics developed biofilm that simulates conditions invivo.

Results show that bacteria are more resistant in biofilm communities as compared to planktonic form by comparison of MIC \& MBEC assay. The higher amount of amikacin, ciprofloxacin, cefoperazone/sulbactam and gentamycin is needed to kill the Klebsiella pneumoniae biofilm than planktonic organism. Ciprofloxacin has good penetration but fails to eradicate biofilms [3]. Shawn et al. (2002), Sepandj et al. (2004) and Nermeen et al. (2011) also concluded that higher amount of amikacin, cefoperazone/sulbactam and gentamycin was needed to kill the Pseudomonas aeruginosa biofilm than planktonic organism [2] [3] [10].

A study by $\mathrm{Qu}$ et al. showed that Biofilm communities of Coagulase negative staphylococci couldn't be 
Table 1. Different clinical bacterial isolates from various indwelling medical devices and analysis of their biofilm production by two different methods.

\begin{tabular}{|c|c|c|c|c|c|c|c|c|}
\hline \multirow[b]{2}{*}{ Organisms } & \multirow[b]{2}{*}{$\begin{array}{c}\text { Endotracheal } \\
\text { Tube }\end{array}$} & \multirow[b]{2}{*}{$\begin{array}{l}\text { Foley's } \\
\text { catheter }\end{array}$} & \multirow[b]{2}{*}{$\begin{array}{l}\text { CVP } \\
\text { Tip }\end{array}$} & \multirow[b]{2}{*}{$\begin{array}{c}\text { Tracheostomy } \\
\text { Tube }\end{array}$} & \multirow[b]{2}{*}{$\begin{array}{l}\text { SPC } \\
\text { Tip }\end{array}$} & \multirow[b]{2}{*}{$\begin{array}{l}\text { Pigtail } \\
\text { Cathter }\end{array}$} & \multicolumn{2}{|c|}{ Biofilm Positive } \\
\hline & & & & & & & $\begin{array}{c}\text { Plate } \\
\text { Method (\%) }\end{array}$ & $\begin{array}{c}\text { Tube } \\
\text { Method (\%) }\end{array}$ \\
\hline Acinetobacterbaumannii & 9 & 1 & 3 & - & - & - & 77 & 54 \\
\hline Klebsiella pneumoniae & 11 & - & - & 1 & - & - & 78 & 67 \\
\hline Pseudomonas aeruginosa & 7 & - & - & 2 & - & - & 45 & 17 \\
\hline Escherichia coli & 3 & 2 & - & - & 1 & - & 75 & 75 \\
\hline Coagulase negative staphylococci & 1 & - & - & - & - & 1 & 100 & 0 \\
\hline Staphylococcus aureus & 1 & - & 1 & - & - & - & 50 & 0 \\
\hline Enterococcus faceium & - & 2 & - & - & - & - & 100 & 0 \\
\hline Proteus mirabilis & 1 & - & - & - & - & - & 0 & 0 \\
\hline Enterobacter cloacae & 1 & - & - & - & - & - & 0 & 0 \\
\hline Total $=50$ & 36 & 5 & 4 & 3 & 1 & 1 & $60 \%$ & $38 \%$ \\
\hline
\end{tabular}

eradicated with higher pharmacological concentrations of gentamicin, oxacillin and vancomycin. Higher amount of antibiotics is needed to kill the Staphylococcus aureus biofilm than planktonic organism. Raphael Saginur et al. (2005) and Farshad et al. (2004) also concluded similar to our study result [3] [4] [10] [11].

\section{Conclusions}

Current antibiotic breakpoints are standardised according to planktonic bacterial form. Patients with medical devices generally work on general infections but colonised by bacteria, new guidelines are required to eradicate biofilm associated bacterial infections. The MBEC assay would be more reliable for selection of clinically effective antibiotics.

Small sample size is the limitation of this study. Further research is needed to add on knowledge and experiences about testing of antibiotic susceptibility of bacterial biofilms and its comparison with other available testing methods. It's a need to find easier methods for diagnosing and quantifying biofilm infection and to develop more specific antimicrobial agents and ideal device surfaces that would surely help to fight against biofilm formation and infections.

\section{References}

[1] Juda, M., Paprota, K., Jałoza, D., Malm, A., Rybojad, P. and Goździuk, K. (2008) EDTA as a Potential Agent Preventing Formation of Staphylococcus epidermidis Biofilm on Polichloride Vinyl Biomaterials. Annals of Agricultural and Environmental Medicine, 15, 237-241.

[2] Nermeen, M.A.A., Shereen, B.E., Manal, M.Y.M. and Ghada, M.E. (2011) Biofilm Forming Bacteria Isolated from Urinary Tract Infection, Relation to Catheterization and Susceptibility to Antibiotics. International Journal for Biotechnology and Molecular Biology Research, 2, 172-178.

[3] Raphael, S., Melissa, D., Wendy, F., Shawn, D.A., Francis, C., Craig, L. and Karam, R. (2006) Multiple Combination Bactericidal Testing of Staphylococcal Biofilms from Implant-Associated Infections. Antimicrobial Agents and Chemotherapy, 50, 55-61. http://dx.doi.org/10.1128/AAC.50.1.55-61.2006

[4] Ceri, H., Olson, M.E., Stremick, C., Read, R.R., Morck, D. and Buret, A. (1999) The Calgary Biofilm Device: New Technology for Rapid Determination of Antibiotic Susceptibilities of Bacterial Biofilms. Journal of Clinical Microbiology, 37, 1771-1776.

[5] Christensen, G.D., Simpson, W.A., Bisno, A.L. and Beachey, E.H. (1982) Adherence of Slime-Producing Strains of Staphylococcus epidermidis to Smooth Surfaces. Infection and Immunity, 37, 318-326.

[6] Bose, S., Khodke, M., Basak, S. and Mallick, S.K. (2009) Detection of Biofilm Producing Staphylococci: Need of the 
Hour. Journal of Clinical and Diagnostic Research, 3, 1915-1920.

[7] Nagaveni, S., Rajeshwari, H., Oli, A.K., Patil, S.A. and Chandrakanth, R.K. (2010) Evaluation of Biofilm Forming Ability of the Multidrug Resistant Pseudomonas aeruginosa. The Bioscan, 5, 563-566.

[8] Revdiwala, S., Rajdev, B. and Mulla, S. (2012) Characterization of Bacterial Etiologic Agents of Biofilm Formationin Medical Devices in Critical Care Setup. Critical Care Research and Practice, 2012, Article ID: 945805. http://dx.doi.org/10.1155/2012/945805

[9] Christensen, G., Simpson, W., Younger, J., Baddour, L., Barret, F., Melton, D. and Beachey, E. (1985) Adherence of Coagulase-Negative Staphylococci to Plastic Tissue Culture Plates: A Quantitative Model for the Adherence of Staphylococci to Medical Devices. Journal of Clinical Microbiology, 22, 996-1006.

[10] Louis, P.G., Howard, C., Allan, P.G., Merle, O. and Farshad, S. (2010) MIC versus MBEC to Determine the Antibiotic Sensitivity of Staphylococcus aureus in Peritoneal Dialysis Peritonitis. Peritoneal Dialysis International, 30, 652-656. http://dx.doi.org/10.3747/pdi.2010.00010

[11] Qu, Y., Istivan, T.S., Daley, A.J., Rouch, D.A. and Deighton, M.A. (2009) Comparison of Various Antimicrobial Agents as Catheter Lock Solutions: Preference for Ethanol in Eradication of Coagulase-Negative Staphylococcal Biofilms. Journal of Medical Microbiology, 58, 442-450. 